Bull. Austral. Math. Soc.

Vol. 52 (1995) [461-474]

\title{
ON THE LIMIT CYCLE DISTRIBUTION OVER TWO NESTS IN QUADRATIC SYSTEMS
}

\author{
Xianhua Huang and J.W. Reyn
}

\begin{abstract}
As a contribution to the solution of Hilbert's 16 th problem the question is considered whether in a quadratic system with two nests of limit cycles at least in one nest there exists precisely one limit cycle. An affirmative answer to this question is given for the case that the sum of the multiplicities of the finite critical points in the system is equal to three.
\end{abstract}

\section{INTRODUCTION}

Of the list of problems enunciated by Hilbert in his address to the International Congress of Mathematicians in Paris in 1900 [9], the sixteenth problem has proved to be a remarkably intractable question. This applies also to its second part, which asks for the maximum number and relative position of the limit cycles of polynomial systems of differential equations in the plane. The problem remains unsolved, even for the simplest case, that of quadratic systems, that is,

$$
\begin{aligned}
& \dot{x}=a_{00}+a_{10} x+a_{01} y+a_{20} x^{2}+a_{11} x y+a_{02} y^{2} \equiv P(x, y), \\
& \dot{y}=b_{00}+b_{10} x+b_{01} y+b_{20} x^{2}+b_{11} x y+b_{02} y^{2} \equiv Q(x, y),
\end{aligned}
$$

where $\equiv \frac{d}{d t}, a_{i j}, b_{i j} \in \mathbb{R}$ and $P(x, y), Q(x, y)$ are relatively prime real polynomials, which are not both linear. In fact, it has only fairly recently been firmly established that a given quadratic system has at most finitely many limit cycles [1], whereas the existence of a uniform upper bound for the number of limit cycles in the class of quadratic systems is still a subject of research [8]. As a result of the work of Bautin [2] and Petrovskii and Landis [12], for some time, an upper bound for the number of limit cycles in quadratic systems was expected to be equal to three until, at the beginning of the eighties, in China, examples with (at least) four limit cycles were given [4, 17]. Since then further examples were given (see for instance $[3,10,13,14,18]$ ). From them and from other results for quadratic systems, the suggestion then emerges that a quadratic system contains at most four limit cycles, in which case they would be distributed over two nests, three in one and one in the other. Since in a quadratic system there can be at

Received 21st February, 1995

Copyright Clearance Centre, Inc. Serial-fee code: 0004-9729/95 \$A2.00+0.00. 
most two nests of limit cycles the question then arises, whether it is generally true that in the case of two nests at least one of them contains precisely one limit cycle. In fact, this property was proved for quadratic systems with four (real) critical points forming a convex quadrangle [20]. In this configuration there are two saddle points and two antisaddles. For the other possible configuration with two nests and four (real) critical points, three antisaddles and one saddle point no proof is available yet. The third possibility with four critical points, that of having two real and two complex critical points, is the one occurring in the examples with (at least) four limit cycles and here again there is no proof, but only evidence. Apart from some limit cases with second order critical points this covers all possibilities, wherein the sum of the multiplicities of the (real or complex) critical points is equal to four. If the sum of the multiplicities of the finite critical points is called the finite multiplicity $m_{f}$ of a quadratic system, this settles $m_{f}=4$. By changing the coefficients in (1), one or more finite critical points may be sent off to infinity yielding $0 \leqslant m_{f}<4$. Trivially for $m_{f}=0$ or 1 no two nests of limit cycles can occur, whereas for $m_{f}=2$ it can be shown that in the case of two nests there is precisely one limit cycle in each nest [15]. This leaves the case $m_{f}=3$; being the case with three finite critical points, possibly complex or coinciding, then yielding a second or third order point. For $m_{f}=3$ a positive answer will be obtained in the present paper. One consequence of this result is that for a bounded quadratic system having two nests of limit cycles at least in one nest there is precisely one limit cycle (and probably in both nests $[7,11]$ ).

For a system with $m_{f}=3$ one critical point has "gone off" to infinity compared to the general quadratic system with four finite critical points. At infinity there thus exists one transversally non-hyperbolic critical point which then necessarily is multiple, whereas possible other infinite critical points are hyperbolic transversal to the Poincaré circle. A multiple infinite critical point in a quadratic system will be indicated by $M_{p, q}^{i}$, where $i$ indicates the index of the point, $p$ the number of finite critical points and $q$ the number of infinite critical points that can at most bifurcate from it upon changing the coefficients in the system. For $m_{f}=3$ thus we have precisely one point $M_{p, q}^{i}$ with $p \neq 0$ and for this point $p=1$. For $q$ there are the possibilities $q=1,2$ or 3 . If $q$ equals 2 or 3 the point $M_{p, q}^{i}$ is a degenerate critical point (for the locally linearised system both eigenvalues are zero), and a result by Coppel [5] shows that the system then has at most one limit cycle; so two nests are impossible. The remaining class in $m_{f}=3$ has an infinite critical point of type $M_{1,1}^{i}$, or as will be shown later, a transversally non-hyperbolic second order saddle node $M_{1,1}^{0}$ with center manifold transversal to the Poincaré circle. In section 2 a normal form will be derived for quadratic systems with finite multiplicity 3 and a $M_{1,1}^{i}$ type of critical point at infinity, and the parameter region indicated for which there are possibly two nests of limit cycles. In section 3 the 
normal form is studied for these parameter regions by transforming it into a Liénard equation. Applying a non-existence and uniqueness theorem for limit cycles of this equation then yields the result that in the case of two nests of limit cycles, in at least one of the nests there is precisely one limit cycle.

\section{QUADRATIC SYSTEMS WITH FINITE MULTIPLICITY 3} AND A $M_{1,1}^{i}$ TYPE OF CRITICAL POINTAT INFINITY

Lemma 1. A quadratic system with finite multiplicity 3 and a $M_{1,1}^{i}$ type of critical point at infinity can be represented by the system

$$
\begin{array}{ll}
\dot{x}=x+\lambda y+\varepsilon y^{2}+\delta(\mu x+\gamma y+x y) & \equiv P(x, y) \\
\dot{y}=\mu x+\gamma y+x y, & \equiv Q(x, y)
\end{array}
$$

where $\mu \geqslant 0, \varepsilon=\mp 1, \lambda, \gamma, \delta \in \mathbb{R}$.

The infinite critical point $M_{1,1}^{i}$ is a transversally non-hyperbolic second order saddle node $M_{1,1}^{0}$ with center manifold transversal to the Poincaré circle.

PRoOF: Critical points of (1) are located at the intersection points of $P(x, y)=0$ and $Q(x, y)=0$, being the $\infty$ and 0 isoclines, respectively, and sending such a point to infinity in a certain direction makes this direction a common asymptotic direction of the conics $P(x, y)=0$ and $Q(x, y)=0$, so that the quadratic terms in (1) have a common linear factor. Without loss of generality this factor may be taken to be $y$, so that $a_{20}=b_{20}=0$ yet, since for $m_{f}=3$ only one critical point is sent to infinity, there is only one common linear factor so $c_{56} \equiv a_{11} b_{02}-a_{02} b_{11} \neq 0$. Since, moreover, an infinite critical point of (1) is in the same direction - given by $y=u x$ - as the flow on the solution curves approaching it, $u$ should be a solution of

$$
f(u)=a_{02} u^{3}+\left(a_{11}-b_{02}\right) u^{2}+\left(a_{20}-b_{11}\right) u-b_{20}=0,
$$

with $a_{20}=b_{20}=0$. For $u=0$ to be a point of first order tangent to the Poincaré circle then $b_{11} \neq 0$. Also it may be seen that for $m_{f}=3$ there exists at least one real critical point in the finite part of the plane which then may be thought to be located in the origin, hence $a_{00}=b_{00}=0$. If, furthermore, $b_{11} x+b_{02} y$ is replaced by $x$, then (1) takes the form

$$
\begin{aligned}
& \dot{x}=a_{10} x+a_{01} y+a_{11} x y+a_{02} y^{2}, \\
& \dot{y}=b_{10} x+b_{01} y+x y,
\end{aligned}
$$

where $a_{02} \neq 0$, since $c_{56}=-a_{02} \neq 0$. It will be convenient to rewrite this equation as

$$
\begin{aligned}
& \dot{x}=a_{10} x+a_{01} y+a_{02} y^{2}+\delta(\mu x+\gamma y+x y), \\
& \dot{y}=\mu x+\gamma y+x y,
\end{aligned}
$$


with $a_{02} \neq 0$ and, since otherwise $m_{f} \leqslant 2, a_{10} \neq 0$. Replacing $x$ by $a_{10} x, y$ by $a_{10} / \sqrt{\left|a_{02}\right|} y$ and $t$ by $t / a_{10}$ then yields

$$
\begin{aligned}
& \dot{x}=x+\lambda y \pm y^{2}+\delta(\mu x+\gamma y+x y), \\
& \dot{y}=\mu x+\gamma y+x y
\end{aligned}
$$

where $\lambda, \delta, \gamma \in \mathbb{R}$ and where we can set $\mu \geqslant 0$, if needed replacing $y$ by $-y$ (then also $\lambda$ and $\delta$ change their sign).

For the determination of the character of $M_{1,1}^{i}$ we may refer to Reyn and Kooij [16]; from figure 2 in [16] it follows with $c_{25}=1 \neq 0$ that $M_{1,1}^{i}$ is a transversally non hyperbolic second order saddle node $M_{1,1}^{0}$ with center manifold transversal to the Poincaré circle.

Possible other finite critical points may be shifted to the origin by means of a translation of the axes leaving system (2) in the same form. In fact, if $\bar{x}=x-x_{c}, \bar{y}=$ $y-y_{c}$, where $\left(x_{c}, y_{c}\right)$ are the coordinates of a finite critical point of $(2)$, this yields

$$
\begin{aligned}
& \dot{\bar{x}}=\bar{x}+\bar{\lambda} \bar{y}+\varepsilon \bar{y}^{2}+\bar{\delta}(\bar{\mu} \bar{x}+\bar{\gamma} \bar{y}+\bar{x} \bar{y}), \\
& \dot{\bar{y}}=\bar{\mu} \bar{x}+\bar{\gamma} \bar{y}+\bar{x} \bar{y}
\end{aligned}
$$

where $\bar{\lambda}=\lambda+2 \varepsilon y_{c}, \bar{\delta}=\delta, \bar{\mu}=\mu+y_{c}$ and $\bar{\gamma}=\gamma+x_{c}$.

For the coordinates $\left(x_{c}, y_{c}\right)$ we have

$$
\begin{aligned}
& x_{c}=-\gamma+\frac{1}{2} \mu\left(\lambda-\varepsilon \mu \pm \sqrt{(\varepsilon \mu+\lambda)^{2}+4 \varepsilon(\gamma-\mu \lambda)}\right) \\
& y_{c}=\frac{1}{2 \varepsilon}\left(-\lambda-\varepsilon \mu \pm \sqrt{(\varepsilon \mu+\lambda)^{2}+4 \varepsilon(\gamma-\mu \lambda)}\right) .
\end{aligned}
$$

We shall use some additional notation to indicate critical points. A finite saddle point is indicated by $e^{-1}$, an antisaddle by $e^{1}$, a complex point by $c^{0}$ and a multiple point by $m_{m}^{i}$, where $i$ indicates the index of the point and $m$ its multiplicity, being equal to 2 or 3 for system (2). Elementary infinite critical points are indicated by $E^{-1}$ for a saddle, $E^{1}$ for an antisaddle (being a node) and $C^{0}$ for a complex point.

Moreover, we shall encounter a tangentially non-hyperbolic second order saddle node which will be indicated by $M_{0,2}^{0}$, using the notation already given.

LEMMA 2. For $\varepsilon=-1$ there are the following combinations of finite critical points: $m_{3}^{1}, m_{2}^{0} e^{1}, e^{-1} e^{1} e^{1}$ and $c^{0} c^{0} e^{1}$ and the following combination of infinite critical points: $C^{0} C^{0} M_{1,1}^{0}$ for $|\delta|<2, M_{0,2}^{0} M_{1,1}^{0}$ for $|\delta|=2$ and $E^{-1} E^{1} M_{1,1}^{0}$ for $|\delta|>2$.

For $\varepsilon=1$ there are the following combinations of finite critical points: $m_{3}^{-1}$, $m_{2}^{0} e^{-1}, e^{-1} e^{-1} e^{1}$ and $c^{0} c^{0} e^{-1}$ and the following combination of infinite critical points: $E^{1} E^{1} M_{1,1}^{0}$. 
ProOF: We study first the critical points at infinity. By using the Poincaré transformation $z=x^{-1}, u=y x^{-1}$, after a scaling of time, (2) becomes

$$
\begin{aligned}
& z^{\prime}=z\left[(1+\mu \delta) z+\delta u+(\lambda+\gamma \delta) u z+\varepsilon u^{2}\right] \equiv R(z, u), \\
& u^{\prime}=-\mu z-u+(1+\delta \mu-\gamma) u z+\delta u^{2}+(\lambda+\gamma \delta) u^{2} z+\varepsilon u^{3} \equiv S(z, u),
\end{aligned}
$$

so that the infinite critical points, apart from $M_{1,1}^{0}$, are located at $z=0$ and the roots of $\varepsilon u^{2}+\delta u-1=0$.

For the product of the eigenvalues in the critical point $\left(0, u_{c}\right)$ we have

$$
\begin{aligned}
\Omega\left(0, u_{c}\right) & =\frac{\partial R}{\partial z}\left(0, u_{c}\right) \frac{\partial S}{\partial u}\left(0, u_{c}\right)-\frac{\partial R}{\partial u}\left(0, u_{c}\right) \frac{\partial S}{\partial z}\left(0, u_{c}\right) \\
& =u_{c}\left(\delta+\varepsilon u_{c}\right)\left(-1+2 \delta u_{c}+3 \varepsilon u_{c}^{2}\right)=2-\delta u_{c} .
\end{aligned}
$$

For $\varepsilon=-1$, if $|\delta|<2$ obviously we have $C^{0} C^{0} M_{1,1}^{0}$ and for $|\delta|=2$ we have $M_{0,2}^{0} M_{1,1}^{0}$. If $|\delta|>2$ there are two elementary points, located in $u_{ \pm}=\left(\delta \pm \sqrt{\delta^{2}-4}\right) / 2$, whereas $\Omega\left(0, u_{+}\right) \Omega\left(0, u_{-}\right)=-\left(\delta^{2}-4\right)<0$ so we have $E^{-1} E^{1} M_{1,1}^{0}$. In all three cases, the sum of the indices of the infinite critical points equals zero. As a result the sum of the indices of the finite critical points is equal to 1 , which yields the possible combinations $m_{3}^{1}, m_{2}^{0} e^{1}, e^{-1} e^{1} e^{1}$ and $c^{0} c^{0} e^{1}$.

For $\varepsilon=1$ we may write $\Omega\left(0, u_{c}\right)=1+u_{c}^{2}>0$, so there are two nodes and there exists only the combination $E^{1} E^{1} M_{1,1}^{0}$ of which the sum of the indices equals 2 , yielding for the sum of the indices of the finite critical points -1 . The finite combinations are then: $m_{3}^{-1}, m_{2}^{0} e^{-1}, e^{-1} e^{-1} e^{1}$ and $c^{0} c^{0} e^{-1}$.

Since in a quadratic system a limit cycle has only one critical point in its interior, this point being an elementary focus, only those combinations containing an antisaddle are of interest for the limit cycle problem. Classes with possibly two nests of limit cycles are $e^{-1} e^{1} e^{1} C^{0} C^{0} M_{1,1}^{0}, e^{-1} e^{1} e^{1} M_{0,2}^{0} M_{1,1}^{0}$ and $e^{-1} e^{1} e^{1} E^{-1} E^{1} M_{1,1}^{0}$. They occur for $\varepsilon=-1$ and this class will now be investigated.

\section{Systems With Possibly tWo NeStS Of Limit CyCles}

We recall that these systems can only be found in $(2)_{e=-1}$ :

$$
\begin{array}{ll}
\dot{x}=x+\lambda y-y^{2}+\delta(\mu x+\gamma y+x y) & \equiv P(x, y), \\
\dot{y}=\mu x+\gamma y+x y & \equiv Q(x, y),
\end{array}
$$

with $\mu \geqslant 0, \lambda, \gamma, \delta \in \mathbb{R}$, and that the coordinates of the finite critical points are given by

$$
\begin{aligned}
& x_{ \pm}=-\gamma+\frac{1}{2} \mu\left(\lambda+\mu \pm \sqrt{(\lambda-\mu)^{2}-4(\gamma-\mu \lambda)}\right) \\
& y_{ \pm}=\frac{1}{2}\left(\lambda-\mu \pm \sqrt{(\lambda-\mu)^{2}-4(\gamma-\mu \lambda)}\right)
\end{aligned}
$$




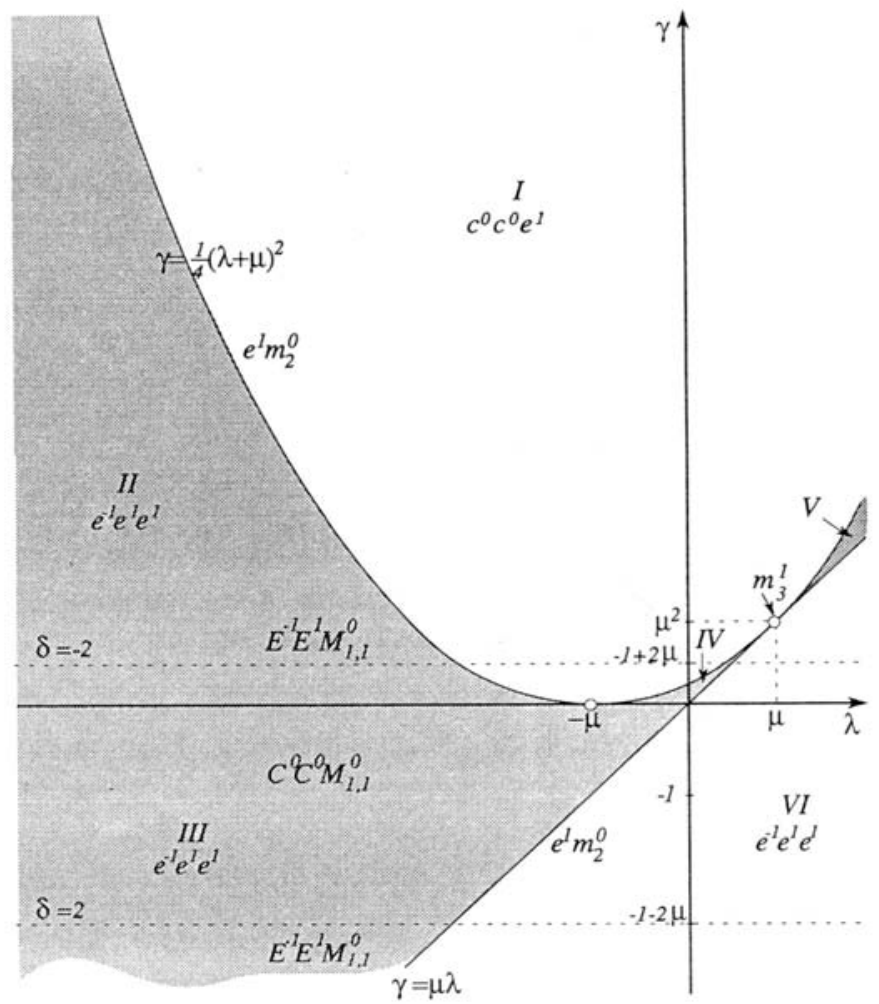

Figure 1. Parameter plane for equation (3).

In figure 1 the $\lambda, \gamma$ parameter plane is given for a given value of $\mu>0$. We do not consider $\mu=0$ since then the system has an invariant line: $y \equiv 0$ and such a quadratic system is known to have at most one limit cycle [6]. In fact the same is true for $\gamma=0$ for which $y \equiv-\mu$ is an invariant line. The various possible combinations of finite critical points are indicated in the corresponding regions. From a local analysis it follows that $(0,0)$ is a saddle for $\gamma-\mu \lambda<0$, an antisaddle for $\gamma-\mu \lambda>0$ and a multiple point for $\gamma-\mu \lambda=0 ; \mathbf{a} m_{3}^{1}$ for $\lambda=\mu, \gamma=\mu^{2}$ and a $m_{2}^{0}$ for $\lambda \neq \mu$. Of interest for the question of two nests of limit cycles is the region $\gamma>\mu \lambda$ where $(0,0)$ is an antisaddle, since if $(0,0)$ is a saddle one of the two antisaddles may be shifted to the origin keeping the same form of equation.

This region may be further subdivided into region I: $-\infty<\lambda<\infty ; \gamma \geqslant$ $(\lambda+\mu)^{2} / 4$, wherein there is only one antisaddle and the regions $\mathrm{II}-\mathrm{V}$ representing systems with two antisaddles. They are given by: II: $-\infty<\lambda<-\mu, 0 \leqslant \gamma<(\lambda+\mu)^{2} / 4$; III: $-\infty<\lambda<0, \mu \lambda<\gamma<0$; IV: $-\mu<\lambda<\mu, \max \{0, \mu \lambda\}<\gamma<(\lambda+\mu)^{2} / 4$ and $\mathrm{V}: \mu<\lambda<\infty, \mu \lambda<\gamma<(\lambda+\mu)^{2} / 4$, and indicated as shaded regions in figure 1. A 
further restriction in the parameter plane $\lambda, \gamma$ is possible since for regions IV and $\mathrm{V}$ at most one nest of limit cycles is possible. We thus arrive at

THEOREM 1. If a quadratic system with finite multiplicity $m_{f}=3$ has two nests of limit cycles, it can be represented by equation (3) with parameter values in the regions $I I\left(-\infty<\lambda<-\mu, 0 \leqslant \gamma<(\lambda+\mu)^{2} / 4\right)$ and $I I I(-\infty<\lambda<0, \mu \lambda<\gamma<0)$.

Proof: If the parameter values are in region $\mathrm{V}, x_{+}>x_{-}, y_{+}>y_{-}>0$ and the situation is sketched in Figure 2. Since $\Omega\left(x_{+}, y_{+}\right)=-2(\gamma-\lambda \mu)+(\lambda-\mu) y_{+}>$ $-2(\gamma-\lambda \mu)+(\lambda-\mu) y_{-}=\Omega\left(x_{-}, y_{-}\right)$, the saddle is at $\left(x_{-}, y_{-}\right)$and the antisaddle at $\left(x_{+}, y_{+}\right)$. If $\left(x_{c}, y_{c}\right)$ are the coordinates of a critical point, from (3) may be obtained that on $y \equiv y_{c}$ we have $\dot{y}=\left(\mu+y_{c}\right)\left(x-x_{c}\right)$, so that with $\mu>0, y_{c} \geqslant 0$ follows that on possible limit cycles around $(0,0)$ and $\left(x_{+}, y_{+}\right)$the motion is in the anticlockwise direction. It is a known result for quadratic systems that this is not possible since the segment of the straight isocline between $(0,0)$ and $\left(x_{+}, y_{+}\right)$must be crossed in the same direction all along this segment.

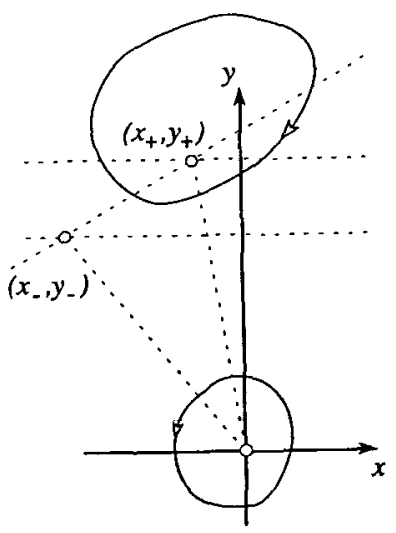

Figure 2.

If the origin is shifted to $\left(x_{+}, y_{+}\right)$; the parameter values are in region IV.

Having focused our attention to regions II and III, it may further be realised that these regions are related to each other through a shift of the origin to the other antisaddle. For either of the two regions it is sufficient then to consider the situation with regard to limit cycles around the origin; the information on limit cycles around the other antisaddle (focus) being taken from the other region. Limit cycles around the origin will be studied by transforming (3) into a Liénard equation. We shall make use of the following lemmas. 
LEMMA 3. Suppose the system

$$
\begin{aligned}
& \dot{x}=-g(y), \\
& \dot{y}=x-F(y),
\end{aligned}
$$

satisfies the following conditions:

(i) $g(y) \in C^{1}$ and $F(y) \in C^{2}$ for $y \in(\alpha, \beta)$, where $\alpha<0<\beta$;

(ii) $y g(y)>0$ for $y \in(\alpha, \beta)$ and $y \neq 0$;

(iii) there exists an $\alpha_{1}$, with $\alpha<\alpha_{1}<0$, such that $f(y)>0$ for $y \in\left(\alpha, \alpha_{1}\right)$ and $f(y)<0$ for $y \in\left(\alpha_{1}, \beta\right)$, where $f(y)=F^{\prime}(y)$;

(iv) $[f(y) / g(y)]^{\prime} \geqslant 0$ for $y \in\left(\alpha, \alpha_{1}\right)$ and $y \in(0, \beta)$.

Then the system has at most one limit cycle in the strip

$$
D_{1}:=\{(x, y) \mid-\infty<x<\infty, \alpha<y<\beta\}
$$

If it exists, it must be a stable cycle, which is hyperbolic.

Suppose, moreover, that the system satisfies conditions (i) and (ii) and in addition the conditions:

(iii)' $f(0)=0, g^{\prime}(0)>0$;

(iv)' $[f(y) / g(y)]^{\prime}>0$ (or $<0$ ) for $y \in(\alpha, \beta)$ and $y \neq 0$.

Then the system has no limit cycle entirely contained in the strip $D_{1}$.

ProOF: As pointed out in [21], the uniqueness part of the theorem follows from Theorem 6.4 of [19] and also from Theorem 1 of [6]. The non-existence part is proved in [21].

NotE. The non-existence part of the lemma is very useful if we want to prove that there is no limit cycle surrounding a weak focus when the sign of the focal values cannot easily be determined.

Lemma 4. System (3) can be transformed into the Liénard system (5), where $f(y)=-f_{1}(y) /(y+\mu)^{2}, g(y)=y g_{1}(y) /(y+\mu)^{2}$ and $f_{1}(y)=\delta y^{2}+(1+2 \mu \delta) y+$ $\mu(\mu \delta+\gamma+1)$ and $g_{1}(y)=y^{2}+(\mu-\lambda) y+\gamma-\lambda \mu$.

PRoOF: In (3), let $x_{1}=\mu x+\gamma y+x y, y_{1}=y$. It then follows that

$$
\begin{aligned}
& \dot{x}_{1}=\frac{1}{\mu+y}\left[-y_{1}^{4}+(\lambda-2 \mu) y_{1}^{3}+\left(2 \mu \lambda-\gamma-\mu^{2}\right) y_{1}^{2}+\mu(\mu \lambda-\gamma) y_{1}\right. \\
& \left.+x_{1}\left\{\delta y_{1}^{2}+(2 \mu \delta+1) y_{1}+\mu(1+\delta \mu+\gamma)\right\}+x_{1}^{2}\right] \\
& \dot{y}_{1}=x_{1} .
\end{aligned}
$$


Then, by $x_{2}=x_{1} /(\mu+y), y_{2}=y_{1}$ and $t_{1}=t(\mu+y)$ we get

$$
\begin{aligned}
& \dot{x}_{2}=\frac{1}{(\mu+y)^{2}}\left[-y_{2}^{3}+(\lambda-\mu) y_{2}^{2}+(\lambda \mu-\gamma) y_{2}\right. \\
& \left.\quad+x_{2}\left\{\delta y_{2}^{2}+(2 \mu \delta+1) y_{2}+\mu(1+\delta \mu+\gamma)\right\}\right] \\
& \dot{y}_{2}=x_{2} .
\end{aligned}
$$

Finally by $x=x_{2}+F\left(y_{2}\right), y=y_{2}$ we obtain (5) with $g(y)$ and $F(y)$, where $f(y)=F^{\prime}(y)$ as stated in the lemma.

As may be seen from (3), $y \equiv-\mu$ is a line without contact which cannot be crossed by a limit cycle. Moreover on $y \equiv y_{ \pm}$we note that $\dot{y}$ is linear in $x$ with the only zero at $\left(x_{ \pm}, y_{ \pm}\right)$. If a limit cycle around the origin crosses this line it has to encircle another critical point as well, which is not possible in a quadratic system.

This indicates the following strips in the $x, y$ plane as regions wherein limit cycles have to be investigated.

$$
\begin{aligned}
& \text { II: }-\infty<\lambda<-\mu, 0 \leqslant \gamma<(\lambda+\mu)^{2} / 4 ; y \in(-\mu, \infty) \\
& \text { III: } \quad-\infty<\lambda<0, \mu \lambda<\gamma<0 ; y \in\left(y_{+}, \infty\right),-\mu<y_{+}<0 .
\end{aligned}
$$

Applying Bendixon's criterion yields the following for region II.

THEOREM 2. For parameter values in region II: $-\infty<\lambda<-\mu, 0 \leqslant \gamma<$ $(\lambda+\mu)^{2} / 4$ equation (3) has no limit cycles around the origin if $\delta \geqslant 0$.

Proof: The roots $f_{1}^{ \pm}$of $f_{1}(y)$ are given by

$$
f_{1}^{ \pm}=\frac{1}{2 \delta}[-(1+2 \mu \delta) \pm \sqrt{1-4 \mu \delta \gamma}]
$$

so $f_{1}^{-}<f_{1}^{+}<0$ for $\gamma \geqslant 0, \delta \geqslant 0(\mu>0)$. In the strip $y \in(-\mu, \infty)$ the divergence of $(-g(y), x-F(y))=-f(y)=f_{1}(y) /(y+\mu)^{2}$ is of constant sign if $f_{1}(y)$ is of constant sign. Sufficient for this is that $f_{1}^{+} \leqslant-\mu$ or $\delta \geqslant 0$.

REMARK. The theorem is equally valid for the regions I, IV and V.

There is no need to obtain a result for $\delta<0$ in region II since complementary information can be obtained from region III.

For region III we apply lemma 3, using lemma 4, where

$$
\left(\frac{f(y)}{g(y)}\right)^{\prime}=-\left(\frac{f_{1}(y)}{y g_{1}(y)}\right)^{\prime}=\frac{\left(y g_{1}(y)\right)^{\prime} f_{1}(y)-f_{1}^{\prime}(y) y g_{1}(y)}{y^{2} g_{1}^{2}(y)}=\frac{\varphi(y)}{y^{2} g_{1}^{2}(y)}
$$

which defines $\varphi(y)$, for which the following lemma is obtained. 
LEMMA 5. If $\delta \geqslant \max \{0,-(\gamma+1) / \mu\}$, for region III $(-\infty<\lambda<0, \mu \lambda<\gamma<0)$ there is $\varphi(y) \geqslant 0$ for $y \in\left(y_{+}, f_{1}^{+}\right) \cup(0, \infty)$.

Proof: First we consider $\underline{\gamma \geqslant-1}$; then $\delta \geqslant 0$. For $\delta=0$ we have

$$
\begin{aligned}
\varphi(y) & =\mu(1+\gamma)(\gamma-\lambda \mu)+2 \mu(1+\gamma)(\mu-\lambda) y+\{\mu-\lambda+3 \mu(1+\gamma)\} y^{2}+2 y^{3} \\
& =\mu(1+\gamma) g_{1}(y)+y\{\mu(1+\gamma)+y\}(-\lambda+\mu+2 y) .
\end{aligned}
$$

From the cubic polynomial we see that, since the coefficients are non-negative, $\varphi(y)>0$ for $y>0$. On the interval $\left(y_{+}, f_{1}^{+}\right)$we have $g_{1}(y)>0, y<0, \mu(1+\gamma)+y<$ 0 and $-\lambda+\mu+2 y>0$ so that $\varphi(y)>0$ on $\left(y_{+}, f_{1}^{+}\right) \cup(0, \infty)$.

For $\delta \neq 0$ we may write

$$
\begin{aligned}
\varphi(y)=\delta y^{4} & +2(1+2 \mu \delta) y^{3}+\{(\mu-\lambda)(1+\mu \delta)+3 \mu(1+\mu \delta+\gamma) \\
& \left.-\delta\left(\gamma-\mu^{2}\right)\right\} y^{2}+2 \mu(\mu-\lambda)(1+\mu \delta+\gamma) y+\mu(\gamma-\lambda \mu)(1+\mu \delta+\gamma)
\end{aligned}
$$

From the quartic polynomial we see, since the coefficients are non negative, that $\varphi(y)>0$ for $y>0$. It remains to consider the interval $y \in\left(y_{+}, f_{1}^{+}\right)$. For fixed values of $\mu, \gamma, \lambda$ we may consider $\varphi(y)$ as a function of $y$ and $\delta$ and use the fact that $\varphi(y, \delta)$ depends linearly on $\delta$. The region in the $y, \delta$ plane on which to consider $\varphi(y, \delta)$ is sketched in figure 3a. Its boundaries are given by $\delta \equiv 0, y \in\left(y_{+},-\mu(1+\gamma)\right) ; y \equiv$ $y_{+}, 0<\delta<\delta\left(f_{1}^{+}\right)$; and the curve $\delta=\delta\left(f_{1}^{+}\right)$. Since $\varphi(y)>0$ for $\delta=0$ and $\varphi(y)$ is linear in $\delta$ it suffices to show that $\varphi\left(f_{1}^{+}\right)>0$ for $\delta=\delta\left(f_{1}^{+}\right)$. This is so since $\varphi\left(f_{1}^{+}\right)=-\left[y g_{1}(y) f_{1}^{\prime}(y)\right]_{y=f_{1}^{+}}$and $y=f_{1}^{+}<0$ for $\delta>0, g_{1}(y)>0$ for $y>y_{+}$and for $f_{1}^{\prime}(y)$ in $y=f_{1}^{+}$we may write $\delta\left(f_{1}^{+}-f_{1}^{-}\right)=\sqrt{1-4 \mu \delta} / 2>0$.

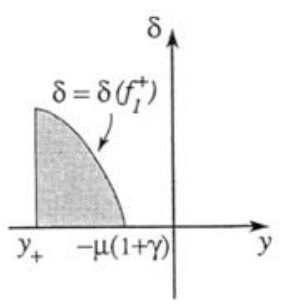

a. $\gamma \geqslant-1$

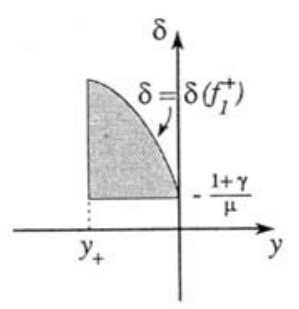

b. $\gamma<-1$

Figure 3. Region in the $y, \delta$ plane where to consider $\phi(y, \delta)$ for region III.

If $\gamma<-1$, then $\delta \geqslant-(\gamma+1) / \mu$ has to be considered. For $\delta=-(\gamma+1) / \mu>0$ from the quartic polynomial (6) it can be seen that, since the coefficients are non negative, $\varphi(y)>0$ for $y>0$. It remains to consider the interval $y \in\left(y_{+}, f_{1}^{+}\right)$. The region in the $y, \delta$ plane on which to consider $\varphi(y, \delta)$ is given in figure $3 \mathrm{~b}$. Its boundaries are given by $\delta=-(\gamma+1) / \mu, y \in\left(y_{+}, 0\right) ; y \equiv y_{+}, \delta>0$; and the curve $\delta=\delta\left(f_{1}^{+}\right)$. 
Since for $\gamma \geqslant-1$ (but not using this condition) it was shown that $\varphi(y)>0$ for $y=f_{1}^{+}$it suffices to show that $\varphi(y) \geqslant 0$ for $\delta=-(\gamma+1) / \mu$. Now (6) yields for $\delta=-(\gamma+1) / \mu$ :

$$
\varphi(y)=y^{2} \psi(y)=y^{2}\left[\delta y^{2}+2(1+2 \mu \delta) y+(\mu-\lambda)(1+\mu \delta)-\delta\left(\gamma-\mu^{2}\right)\right],
$$

where $\psi(y)$ takes its minimum at $y=-1 / \delta(1+2 \mu \delta)<-\mu<y_{+}$so it is sufficient to show that $\psi\left(y_{+}\right)>0$. However, $\varphi\left(y_{+}\right)=\left[\left(y g_{1}(y)\right)^{\prime} f_{1}(y)\right]_{y=y_{+}}=$ $\delta y_{+}\left(y_{+}-y_{-}\right)\left(y_{+}-f_{1}^{+}\right)\left(y_{+}-f_{1}^{-}\right)>0$, thus $\psi\left(y_{+}\right)>0$.

We can now state the following theorem.

TheOREM 3. For region III $(-\infty<\lambda<0, \mu \lambda<\gamma<0)$ there is at most one limit cycle around the origin for equation (3) if $\delta \geqslant \max \{0,-(\gamma+1) / \mu\}$. If it exists, it is a stable cycle, which is hyperbolic.

Proof: Apply lemma 3, using lemma 4 and 5 and putting $\alpha=y_{+}, \beta=\infty$ and $\alpha_{1}=f_{1}^{+}$.

For region III lemma 3 may further be applied to complete the information for $\delta>0$.

Theorem 4. For region III $(-\infty<\lambda<0, \mu \lambda<\gamma<0)$ there is no limit cycle around the origin for equation (3) if $\delta \leqslant-(\gamma+1) / \mu, \gamma \leqslant-1$.

Proof: For $\delta=-(\gamma+1) / \mu$, the origin is a weak focus for (3). From the non existence part of Lemma 3 it follows that there is no limit cycle around the weak focus. In fact conditions (i) and (ii) are equally satisfied putting $\alpha=y_{+}, \beta=\infty$, whereas, clearly $f(0)=0, g^{\prime}(0)=1 / \mu^{2}(\gamma-\lambda \mu)>0$ and according to the proof of lemma 5 $[f(y) / g(y)]^{\prime}>0$ for $y \in\left(y_{+}, \infty\right)$ and $y \neq 0$ as $f_{1}^{+}=0$ for $1+\gamma+\mu \delta=0$. The same conclusion may also be obtained by considering the change of stability of the focus in the origin when $\delta$ is varied through $\delta=-(\gamma+1) / \mu$ and using Theorem 3. To determine its stability, first replace $\mu x+\gamma y$ by $x$ in (3) to obtain

$$
\begin{aligned}
& \dot{x}=(1+\mu \delta+\gamma) x-(\gamma-\lambda \mu) y+\left(\delta+\frac{\gamma}{\mu}\right) x y-\frac{1}{\mu}\left(\mu^{2}+\mu \delta \gamma+\gamma^{2}\right) y^{2}, \\
& \dot{y}=x+\frac{1}{\mu} x y-\frac{\gamma}{\mu} y^{2},
\end{aligned}
$$

whereas replacing $x$ by $\mu(\gamma-\lambda \mu) x, y$ by $\mu y \sqrt{\gamma-\lambda \mu}$ and $t$ by $t / \sqrt{\gamma-\lambda \mu}$ leads to

$$
\begin{aligned}
& \dot{x}=\frac{1+\gamma+\mu \delta}{\sqrt{\gamma-\lambda \mu}} x-y+(\gamma+\mu \delta) x y-\frac{\mu^{2}+\mu \gamma \delta+\gamma^{2}}{\sqrt{\gamma-\lambda \mu}} y^{2}, \\
& \dot{y}=x+\sqrt{\gamma-\lambda \mu} x y-\gamma y^{2} .
\end{aligned}
$$


For the first focal value $W_{1}$ we have, see [19],

$$
W_{1}=\frac{1}{\mu^{2} \sqrt{\gamma-\lambda \mu}}\left[\mu^{2}+\left(-1+2 \mu^{2}-\mu \lambda\right) \gamma-\gamma^{2}\right]
$$

and $W_{1}<0$ for $\gamma \leqslant-1$ in region III, and the weak focus is stable. Since for $\delta>$ $-(1+\gamma) / \mu$, the origin is an unstable strong focus, precisely one (stable) limit cycle, which is hyperbolic will be bifurcated in a Hopf bifurcation, and since, according to Theorem 3 there is only one limit cycle, there will be none around the weak focus. Now consider the Poincaré mapping around the origin for (3).

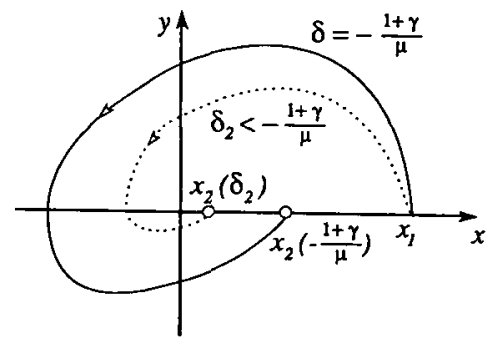

Figure 4.

As illustrated in figure 4 a path intersecting the positive $x$ axis at $x=x_{1}$ again intersects at $x=x_{2}$ after one time wise (anticlockwise) revolution around the origin. For the (stable) weak focus without limit cycle around it $x_{2}=x_{2}\left(x_{1} ; \delta\right)<x_{1}$ for $x_{1}>0$. For $\delta<-(1+\gamma) / \mu$ the path through the same value of $x_{1}$ spirals in more strongly as may be seen from the expression

$$
\left|\begin{array}{cc}
P & Q \\
\frac{\partial P}{\partial \delta} & \frac{\partial Q}{\partial \delta}
\end{array}\right|=-Q^{2} \leqslant 0 .
$$

As a result for $\delta \leqslant-(1+\gamma) / \mu, \gamma \leqslant-1, x_{2}=x_{2}\left(x_{1} ; \delta\right)<x_{1}$ for $x_{1}>0$ and there is no limit cycle around the origin.

Having obtained the focal value $W_{1}$ for the weak focus in the origin, figure 1 may also be viewed as the projection of the fine focus plane $1+\mu \delta+\gamma=0$ in the $\lambda, \gamma, \delta$ space onto the $\lambda, \gamma$ plane, wherein the value of $W_{1}$ may be indicated.

\section{Distribution of limit CyCles OVER TWo Nests}

Now we can state

THEOREM 5. If a quadratic system with finite multiplicity $m_{f}=3$ has two nests of limit cycles, at least one nest contains precisely one limit cycle.

Proof: From theorems 3 and 4 follows for region III that there is at most one limit cycle around the origin if $\delta \geqslant 0$. If $\delta<0$ there is at most one nest of limit cycles, 
since around the antisaddle not in the origin there exists no limit cycle. This may be derived from the result for region II in Theorem 2 that for $\delta \geqslant 0$ there does not exist a limit cycle around the origin. In order to demonstrate this, the origin will be shifted to the other antisaddle, which is located at $\left(x_{-}, y_{-}\right)$, the saddle being located at $\left(x_{+}, y_{+}\right)$. Using the transformation $\bar{x}=x-x_{-}, \bar{y}=y-y_{-}$on (3) yields

$$
\begin{aligned}
& \dot{\bar{x}}=\bar{x}+\bar{\lambda} \bar{y}-\bar{y}^{2}+\bar{\delta}(\bar{\mu} \bar{x}+\bar{\gamma} \bar{y}+\bar{x} \bar{y}), \\
& \dot{\bar{y}}=\bar{\mu} \bar{x}+\bar{\gamma} \bar{y}+\bar{x} \bar{y}
\end{aligned}
$$

where $\bar{\lambda}=\lambda-2 y_{-}, \bar{\delta}=\delta, \bar{\mu}=\mu+y_{-}$and $\bar{\gamma}=\gamma+x_{-}$.

Using (4) this gives $\bar{\mu}=(\lambda+\mu) / 2-\sqrt{(\lambda-\mu)^{2}-4(\gamma-\mu \lambda)} / 2<0$, since $\lambda+\mu<0$ in region II. Replacing $y$ by $-y$ now leads to a system with $\mu_{1}=-\bar{\mu}>0, \lambda_{1}=$ $-\bar{\lambda}, \delta_{1}=-\bar{\delta}$ and $\bar{\gamma}_{1}=\bar{\gamma}$. It then follows that

$$
\begin{aligned}
0>\gamma_{1} & =1 / 2 \mu\left(\lambda+\mu-\sqrt{(\lambda-\mu)^{2}-4(\gamma-\mu \lambda)}\right) \\
& >\frac{1}{2}\left(\mu+\sqrt{(\lambda-\mu)^{2}-4(\gamma-\mu \lambda)}\right)\left(\lambda+\mu-\sqrt{(\lambda-\mu)^{2}-4(\gamma-\mu \lambda)}\right) \\
& =\mu_{1} \lambda_{1}
\end{aligned}
$$

and the mapping is into region III with $\delta_{1}>0$.

\section{REFERENCES}

[1] R. Bamón, 'Quadratic vector fields in the plane have a finite number of limit cycles', Inst. Hautes Etudes Sci. Publ. Math. 64 (1986), 111-142.

[2] N.N. Bautin, 'On the number of limit cycles which appear with the variation of coefficients from an equilibrium position of focus or centre type', (in Russian), Mat. Sb. 30 (1952), 181-196, Amer. Math. Soc. Transl. no. 100 (1954), 396-413; reprint AMS Transl. 5, (1962), 396-413.

[3] T.R. Blows and N.G. Lloyd, 'The number of limit cycles of certain polynomial differential equations', Proc. Roy. Soc. Edinburgh Sect. A (1984), 215-239.

[4] Chen Lansun and Wang Mingshu, 'Relative position and number of limit cycles of a quadratic differential system', (in Chinese), Acta Math. Sinica 22 (1979), 751-758.

[5] W.A. Coppel, 'Quadratic systems with a degenerate critical point', Bull. Austr. Math. Soc. 38 (1988), 1-10.

[6] W.A. Coppel, 'Some quadratic systems with at most one limit cycle', Dynam. Reported 2 (1989), 61-88.

[7] R.J. Dickson and L.M. Perko, 'Bounded quadratic systems in the plane', J. Differential Equations 7 (1970), 251-273. 
[8] F. Dumortier, R. Roussarie and C. Rousseau, 'Hilbert's 16 th problem for quadratic vector fields', J. Differential Equations 110 (1994), 86-133.

[9] D. Hilbert, 'Mathematische Probleme', Lecture, Second Internat. Congr. Math. (Paris, 1900), Nachr. Ges. Wiss. Göttingen Math.-Phys. Kl. (1900), 253-297; reprinted in Arch. Math. Phys. 1 (1901), 44-63, 213-237 and also Gesammelte Abhandlungen. Vol. III, 2nd ed., Springer-Verlag, 1970, pp. 290-329; English trans. Bull. Amer. Math. Soc. 8 (1902), 437-479; reprinted in Mathematical Developments Arising from Hilbert Problems, Proc. Symp. Pure Math., 28, (Amer. Math. Soc., Providence, R.I.), 1976, pp. 1-34.

[10] Li Chengzhi, 'Two problems of planar quadratic systems,', Sci. Sinica A26 (1983), 471-481.

[11] L.M. Perko, Coppel's problem for bounded quadratic systems (Dep. of Math., Northern Arizona University, Flagstaff).

[12] I.G. Petrovskii and E.M. Landis, 'On the number of limit cycles of the equation $\frac{d y}{d x}=\frac{P(x, y)}{Q(x, y)}$, where $P$ and $Q$ are polynomials of 2nd degree', Mat. Sb. 37 (1955), 209-250.

[13] Qin Yuanxun, Shi Songling and Cai Suilin, 'On limit cycles of planar quadratic systems', Sci. Sinica A25 (1982), 41-50.

[14] Qin Yuanxun, Shi Songling and Cai Suilin, 'On limit cycles of planar quadratic systems', Sci. Sinica A26 (1983), 1025-1033.

[15] J.W. Reyn and R.E. Kooij, 'Phase portraits of quadratic systems with finite multiplicity two', (preprint).

[16] J.W. Reyn and R.E. Kooij, 'Infinite singular points of quadratic systems in the plane', $J$. Nonlinear Analysis: Theory, Methods and Applications 24 (1995), 895-927.

[17] Shi Songling, 'A concrete example of the existence of four limit cycles for plane quadratic systems', Scientia Sinica 23, 153-158.

[18] G. Wanner, 'Über Shi's Gegenbeispiel zum 16. Hilbertproblem', Jahrbuch Überblicke Mathematik (1983), 9-24.

[19] Ye Yan-Qian and others, Theory of limit cycles, Translations of Mathematical Monographs 66 (Amer. Math. Soc., Providence, R.I., 1986).

[20] Zhang Pingguang, 'On the concentrative distribution and uniqueness of limit cycles for a quadratic system', (Proc. of the Special Program at the Nakai Inst. of Math., Tianjin, P.R. China (Sept. 1990-June 1991)), in Nakai Series in Pure, Appl. Math. and Theor. Phys., Dynamical Systems 4, pp. 297-310.

[21] Zhang Pingguang and Cai Suilin, 'Quadratic systems with a weak focus', Bull. Austral. Math. Soc. 44 (1991), 511-526.

\footnotetext{
Faculty of Technical Mathematics and Informatics

Delft University of Technology

PO Box 5031

2600 GA Delft

The Netherlands
} 